SHORT COMMUNICATION

\title{
Pseudomonas aeruginosa Infection in African Grey Parrot and its Antibiogram Study
}

\author{
Shailja Katoch*1, Jeny K John², Akshay Garg' ${ }^{1}$ and Rajeev Singh ${ }^{1}$ \\ ${ }^{1}$ Department of Veterinary Microbiology, COVAS, S. V. P. University of Agriculture E Technology, Meerut, INDIA \\ ${ }^{2}$ Department of Teaching Veterinary Clinical Complex, COVAS, S. V. P. University of Agriculture E Technology, Meerut, INDIA
}

*Corresponding author: S Katoch; E-mail: dr.shailjakatoch@gmail.com

Received: 01 July, 2020

Revised: 18 Aug., 2020

Accepted: 29 Aug., 2020

\begin{abstract}
Case study of Pseudomonas aeruginosa infection in a seven years old, male African grey parrot reared in aviary in Meerut, died 48 hours after exhibiting clinical signs is reported. The gross examination of carcass revealed heavy ectoparasitic infestation and necropsy examination revealed inflammed respiratory tract, airsaculitis and congestion of liver, spleen and intestine. Microbiological examination of the tissue samples revealed Pseudomona aeruginosa infection, which was found susceptible to quinolones i.e. ciprofloxacin and enrofloxacin, intermediately susceptible to aminoglycosides i.e. gentamicin and streptomycin, but resistant to cephalosporins i.e. ceftriaxone and cefotaxime/clavulanic acid, $\beta$-lactam/ $\beta$-lactamase inhibitor combinations i.e. amoxicillin/clavulanic acid and ampicillin/cloxacillin, tetracyclines i.e. oxytetracycline, macrolides i.e. erythromycin and chloramphenicol in culture sensitivity test. These antibiotic resistant bacteria can pose threat to in contact immunocompromised aviary workers. Thus necessary biosecurity measures should be implemented in the aviaries to avoid environment associated infectious diseases.
\end{abstract}

\section{HIGHLIGHTS}

( Pseudomonas aeruginosa infection in African grey parrot

(- Ectoparasitic infestation, lesions on respiratory tract and visceral organs of carcass

( Pseudomonas aeruginosa exhibited antibiotic resistance to commonly used antibiotics

Keywords: Pseudomonas aeruginosa, African grey parrot, Antibiotic resistance

The Pseudomonas aeruginosa is a gram-negative, motile, non-spore forming obligate aerobic rod which produces large, flat, serrated, green or blue pigmented colonies having characteristic sweetish fruity odour (Quinn et al., 2011). The organism is ubiquitous and occurs widely in soil, water, feed and humid conditions as environmental infectious agent and is resistant to various antibiotics and commonly used disinfectants.

It is a common opportunistic avian pathogen causing both localized infections in the upper respiratory tract such as rhinitis, sinusitis and laryngitis as well as septicaemic infections (Gerlach, 1994; Momotani et al., 1995). There are previous reports of septicaemia and haemorrhagic enteritis in psittacine birds, corneal ulcers in captive cranes (Miller et al., 1995) and mass mortality in freeliving flamingos (Kock and Kock, 1995). The present case study reports the isolation of $P$. aeruginosa from African grey parrot carcass and its antibiotic sensitivity profile.

African grey parrot carcass was presented at department of Teaching Veterinary Clinical Complex (TVCC) of College of Veterinary \& Animal Sciences, SVPUA\&T, Meerut for necropsy. The history of case was recorded and necropsy was conducted. The samples of heart blood, trachea, liver, spleen and intestine were collected

How to cite this article: Katoch, S., John, J.K., Garg, A. and Singh, R. (2020). Pseudomonas aeruginosa infection in African grey parrot and its antibiogram study. J. Anim. Res., 10(5): 849-852.

Source of Support: None; Conflict of Interest: None (क) 
aseptically and submitted to department of Veterinary Microbiology, for identification of causative agent (s) and their culture sensitivity test. The samples were inoculated on Nutrient agar and Maconkey's Lactose agar and incubated at $37^{\circ} \mathrm{C}$ for $24-48$ hours. The organism was identified on the basis of primary and secondary biochemical tests. The in-vitro culture sensitivity test was carried out using the Kirby- Bauer disc diffusion method described by Bailey et al. (1998) on Mueller Hinton Agar plate using antibiotic discs of Gentamicin, Streptomycin, Amoxicillin/Clavulanic acid, Ampicillin/ Cloxacillin, Oxytetracycline, Ciprofloxacin, Enrofloxacin, Chloramphenicol, Ceftriaxone, Cefotaxime/Clavulanic acid and Erythromycin. The plate was incubated at $37^{\circ} \mathrm{C}$ for 18 hours and susceptibility to antimicrobials was recorded by measuring the diameter of zone of inhibition (NCCLS, 2004).

Pseudomonas is one amongst the most common pathogens, associated with upper respiratory tract diseases in birds and causes outbreaks in the aviary by contamination of water and feed with organic material (Fudge et al., 1992; Jackson; Phillips, 1996; Reavill, 1996). The susceptibility of birds to Pseudomonas aeruginosa infection depends upon nutritional status, immune resistance, concurrent infections (Barnes, 1997), transportation ((Momotani et al., 1996) and age (Miller et al., 1995). The role of environmental factors, nutritional deficiencies and inappropriate use of antibiotics as precipitating factors in upper respiratory tract disease in psittacines has already been reported by Tully and Harrison (1994). The present case report, revealed the death of seven year old male African grey parrot housed in an aviary after exhibiting the clinical signs of dullness, inappetence, polydipsia, yellow urine, nasal and ocular discharges for 2 days. The physical examination of the carcass showed matting of loreal and facial feathers due to ocular and nasal discharge and heavy ectoparasitic infestation. The ectoparasitic infestation may have caused the decrease in host defense system predisposing the bird to prevailing Pseudomonas aeruginosa infection.

The gross pathological examination revealed rhinitis, sinusitis, tracheitis and air saculitis. The serosal membranes showed fibrinous inflammation. The liver, spleen and intestines were congested. Liver showed multiple necrotic foci randomly distributed over the liver parenchyma. The present findings are in agreement with previous reports of outbreaks by Pseudomonas species in aviary (Gerlach, 1994).

The culture of samples showed large, flat, serrated and greenish pigmented colonies with fruity grape like odour on nutrient agar plate (Fig. 1). On Maconkey's Lactose agar large, pale colonies of non-lactose fermenter with greenish pigmentation were observed. The organism was motile, medium sized Gram negative rod, gave positive reactions to oxidase, catalase, arginine dehydrolase, urease, nitrate reduction, gelatin liquification and citrate utilization test. Triple Sugar Iron agar slant remained unchanged. Negative reactions were recorded for lysine decarboxylase and ornithine decarboxylase test. The isolate oxidized glucose but not lactose and sucrose. The results of biochemical tests on the isolate of present investigation are in agreement to Quinn et al. (2011) and confirm the isolate as Pseudomonas aeruginosa.

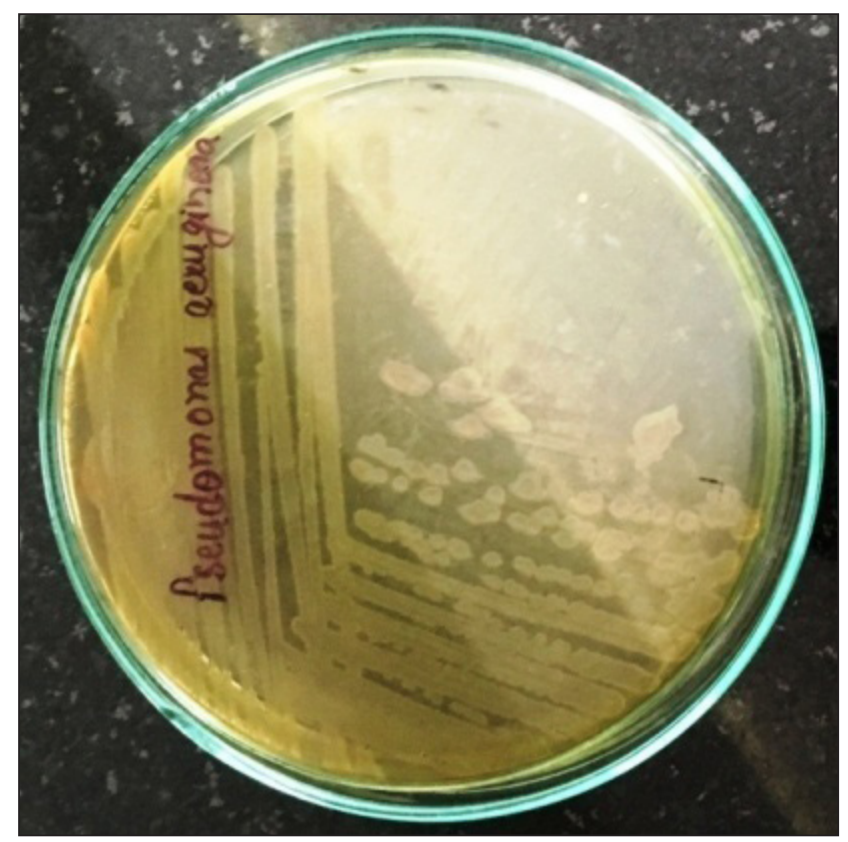

Fig. 1: Flat, serrated and greenish colonies of Pseudomonas aeruginosa on Nutrient Agar plate

The antibiotic sensitivity test revealed that the organism was found susceptible to quinolones i.e. ciprofloxacin and enrofloxacin, intermediately susceptible to aminoglycosides i.e. gentamicin and streptomycin, but resistant to cephalosporins i.e. ceftriaxone and cefotaxime/clavulanic acid, $\beta$-lactam/ $\beta$-lactamase inhibitor combinations i.e. amoxicillin/clavulanic acid and 
ampicillin/cloxacillin, tetracyclines i.e. oxytetracycline, macrolides i.e. erythromycin and chloramphenicol (Fig. 2). The previous investigations had also reported resistance in organism to most commonly used antibiotics and susceptibility to aminoglycosides and quinolones (Fudge et al.,1992; Reavill, 1996; Kebede, 2010) and had recommended combination therapy for treatment of Pseudomonas infection in birds.

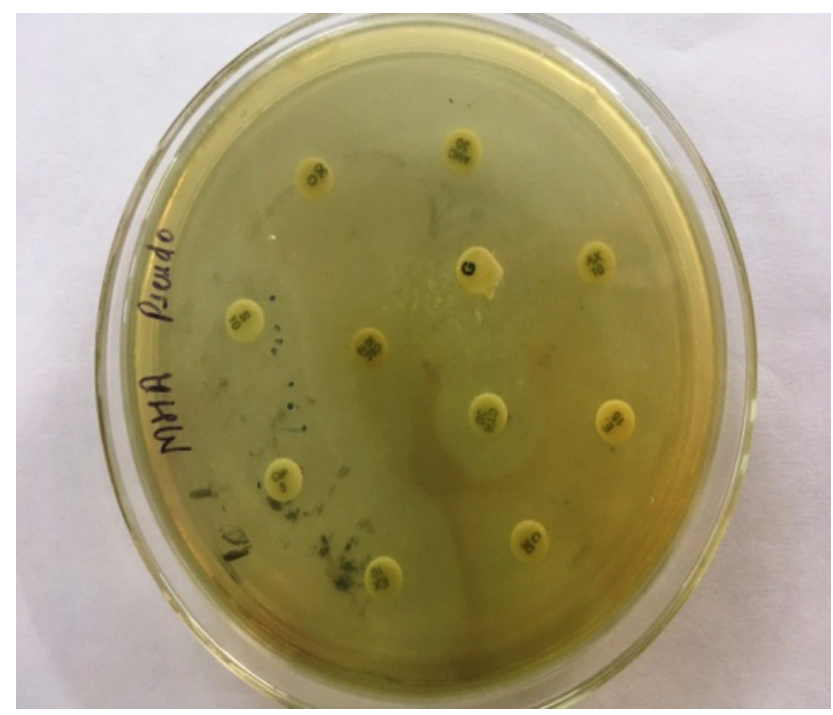

Fig. 2: Culture sensitivity test of the $P$. aeruginosa on Muller Hinton Agar plate

\section{CONCLUSION}

The study indicates that antibiotic resistant Pseudomonas aeruginosa is circulating in birds and may pose threat to in contact immunocompromised aviary workers. Therefore, stringent measures of regular cleaning at farm along with periodic checks for the presence of pathogenic environment contanimants may be enforced to prevent the Pseudomonas infection in the aviaries. The workers should be well trained on how to avoid environmental associated infectious diseases and in implementing all the necessary biosecurity measures.

\section{ACKNOWLEDGEMENTS}

The authors thank the Hon'ble Vice-Chancellor \& Dean, College of Veterinary \& Animal Sciences, Sardar Vallabhbhai Patel University of Agriculture \& Technology, Meerut for providing necessary facilities of work.

\section{REFERENCES}

Bailey, T.A., Silvanose, C.D., Wernery, U., Samour, J.H. and Naldo, J. 1998. Antimicrobial resistance and minimum inhibitory concentrations of bacteria isolated from bustards in the United Arab Emirates. Avian Dis., 42: 690-697.

Barnes, J.H. 1997. Other Bacterial Diseases. In: Diseases of Poultry. Calnek, B.W. (Ed), $10^{\text {th }}$ edn., Iowa State University Press, Ames, IA, USA, pp. 291-292.

Fudge, A.M., Reavill, D.R. and Rosskopf, W.J. 1992. Clinical aspects of avian Pseudomonas infections: A retrospective study. In: Proceedings of the Association of Avian Veterinarians. September, 1992. New Orleans, Louisiana, USA, pp. 141-155.

Gerlach, H. 1994. Bacteria. In: Avian medicine principles and application. Ritchie, B.W., Harrison, G.J. and Harrison, L.R. (Eds), Wingers, Publishing Inc., Lake Worth, Florida, USA, pp. $950-983$.

Jackson, K. and Phillips, S.N. 1996. Necrotizing hepatitis in pet birds associated with Pseudomonas fluorescens. Avian Dis., 40(2): 473-476.

Kebede, F. 2010. Pseudomonas infection in chickens. J. Vet. Med. Anim. Health, 2(4): 55-58.

Kock, N. and Kock, R. 1995. Disease epidemic in lesser flamingos (Phoeniconauis minor) in Kenya. In: Proceedings of the American Association of Zoo Veterinarians, American Association of Zoo Veterinarians. August 1995. East Lansing, Michigan, USA, pp. 115.

Miller, P.E., Langenberg, J.A., Baeten, L.A. and Moore, C.P. 1995. Pseudomonas aeruginosa associated corneal ulcers in captivecranes. J. Avian Med. Surg., 9(4): 283-287.

Momotani, E., Kiryu, M., Ohshiro, M., Murakami, Y., Ashida, Y., Watanabe, S. and Matsubara, Y. 1995. Granulomatous lesions caused by Pseudomonas aeruginosa in the Ostrich (Struthio camelus). J. Comp. Pathol., 112(3): 273-282.

NCCLS, 2004. Performance standards for antimicrobial disk and dilution susceptibility tests for bacteria isolated from animals. Approved standard M31-A2. $2^{\text {nd }}$ Ed., National Committee for Clinical Laboratory Standards, Wayne, Pennsylvania.

Quinn, P.J., Markey, B.K., Fitz Patrick, E.S., Fanning, S. and Hartigan, P.J. 2011. Pseudomonas aeruginosa and Burkholderia species. In: Veterinary Microbiology and Microbial Disease, $2^{\text {nd }}$ edn, Willey Blackwell, UK, pp. 287292. 
Reavill, D. 1996. Bacterial diseases. In: Diseases of cage and aviary birds. Rosskopf, W. and Woerpel, R. (Eds.), $3^{\text {rd }}$ edn,Williams and Wilkins, Baltimore, Maryland, USA, pp. 596-612.
Tully, T.N. and Harrison, G.J. 1994. Pneumology. In: Avian medicine: principles and application, Ritchie, B.W., Harrison, G.J. and Harrison, L.R. (Eds.), Wingers, Publishing Inc., Lake Worth, Florida, USA, pp. 556-581. 